\title{
Short-term influence of cataract surgery on circadian biological rhythm and related health outcomes (CLOCK-IOL trial): study protocol for a randomized controlled trial
}

Keigo Saeki ${ }^{*}$, Kenji Obayashi ${ }^{1}$, Tomo Nishi ${ }^{2}$,Kimie Miyata ${ }^{2}$, Shinji Maruoka ${ }^{2}$, Tetsuo Ueda ${ }^{2}$, Masahiro Okamoto ${ }^{2}$, Taiji Hasegawa ${ }^{2}$, Toyoaki Matsuura ${ }^{2}$, Nobuhiro Tone ${ }^{3}$, Nahoko Ogata ${ }^{2}$ and Norio Kurumatani ${ }^{1}$

\begin{abstract}
Background: Light information is the most important cue of circadian rhythm which synchronizes biological rhythm with external environment. Circadian misalignment of biological rhythm and external environment is associated with increased risk of depression, insomnia, obesity, diabetes, cardiovascular disease, and cancer. Increased light transmission by cataract surgery may improve circadian misalignment and related health outcomes. Although some observational studies have shown improvement of depression and insomnia after cataract surgery, randomized controlled trials are lacking. We will conduct a parallel-group, assessor-blinded, simple randomized controlled study comparing a cataract surgery group at three months after surgery with a control group to determine whether cataract surgery improves depressive symptoms, sleep quality, body mass regulation, and glucose and lipid metabolism.

Methods/Design: We will recruit patients who are aged 60 years and over, scheduled to receive their first cataract surgery, and have grade 2 or higher nuclear opacification as defined by the lens opacities classification system III. Exclusion criteria will be patients with major depression, severe corneal opacity, severe glaucoma, vitreous haemorrhage, proliferative diabetic retinopathy, macular oedema, age-related macular degeneration, and patients needing immediate or combined cataract surgery. After baseline participants will be randomized to two groups. Outcomes will be measured at three months after surgery among the intervention group, and three months after baseline among the control group. We will assess depressive symptoms as a primary outcome, using the short version geriatric depression scale (GDS-15). Secondary outcomes will be subjective and actigraph-measured sleep quality, sleepiness, glycated haemoglobin, fasting plasma glucose and triglyceride, low-density lipoprotein cholesterol, high-density lipoprotein cholesterol, body mass index, abdominal circumference, circadian rhythms of physical activity and wrist skin temperature, and urinary melatonin metabolite. Chronotype and visual function will be assessed using the 'morningness-eveningness' questionnaire, the Munich chronotype questionnaire, and the National Eye Institute Visual Function Questionnaire.

(Continued on next page)
\end{abstract}

\footnotetext{
*Correspondence: saekik@naramed-u.ac.jp

${ }^{1}$ Department of Community Health and Epidemiology, Nara Medical

University School of Medicine, 840 Shijocho, Kashiharashi, Nara 634-8521,

Japan

Full list of author information is available at the end of the article
} 
(Continued from previous page)

Discussion: Although there are potential limitations due to the difference in duration from baseline survey to outcome measurements between two groups, any seasonal effect on the outcome measurement will be balanced as a result of continuous inclusion of participants through the year, and outcomes will be adjusted for day length at outcome measurements at analysis.

Trial registration: UMIN000014559, UMIN Clinical Trials Registry, registered on 15 July 2014.

Keywords: Cataract surgery, Circadian rhythm, Depression, Sleep disturbance, Diabetes mellitus

\section{Background}

Light information is the dominant stimulus synchronising the master internal biological clock at the suprachiasmatic nuclei $(\mathrm{SCN})$ with the external environment. The internal biological rhythm of humans is close to that of the earth's rotation, according to a study of the rhythm of core body temperature independent of the external environment [1]. The misalignment of biological rhythm and external environment is associated with many health problems. Epidemiological studies among shift workers suggest that circadian misalignment is significantly associated with an increased risk of sleep disturbance [2], depression [3], obesity and metabolic syndrome [4,5], diabetes [6,7], ischaemic heart disease [8,9], and stroke [10]. Light information modifies the timing of the internal biological rhythm according to the phase response curve to light [11]. Light exposure in the early morning (after core body temperature minimum) is responsible for the subsequent phase advance in melatonin secretion; in contrast, light in the evening (before core body temperature minimum) is responsible for the subsequent phase delay. Simultaneously, the amplitude of the internal biological rhythm is modified by light information [12].

In contrast to visual light information received by rod and cone cells of the retina and transmitted via the optic nerve, non-visual light information is mainly perceived by recently discovered intrinsically photosensitive retinal ganglion cells (ipRGCs), which contain melanopsin, and are transmitted to the $\mathrm{SCN}$ via the retinohypothalamic tract (RHT) [13]. The action spectrum for melatonin suppression among humans show a peak at a shorter wavelength $(464 \mathrm{~nm})$ than that for visual information (approximately $555 \mathrm{~nm})$ [14].

Cataracts are a prevalent cause of visual impairment and a leading cause of blindness worldwide. The World Health Organisation reported that $33 \%$ of visual impairment (representing 95 million people) and 51\% of blindness (representing 19.9 million people) are due to cataracts [15]. Among age-related cataract patients, the light transmission at the most sensitive spectrum for the photic entrainment of internal biological rhythms decreases from $82 \%$ at 10 years to $23 \%$ at 80 years [16]. Circadian misalignment because of the decreased input of light information caused by cataracts may explain the higher prevalence of depression among cataract patients [17], and the association of decreased light transmission by lens yellowing with sleep disturbance [18].

The research hypothesis of the present study is that cataract surgery, which removes the clouded lens and implants an artificial intraocular lens (IOL), will increase the input of non-visual light information and improve circadian alignment and its related health outcomes, such as depression, sleep disturbances, body mass regulation, and glucose and lipid metabolism [19].

The hypothesis is supported by studies of bright light intervention and light exposure in real-life situations. A Cochrane systematic review showed a significant reduction of depressive symptoms (standardised mean difference -0.20 , $95 \%$ CI -0.38 to -0.01 ) from a metaanalysis of 18 randomized controlled trials (RCTs) among 505 patients with non-seasonal depression [20], and another systematic review also revealed the significant effectiveness of bright light intervention and light exposure among seasonal affective disorders (eight RCTs totalling 132 patients) [21]. In addition, recent RCTs have revealed the effectiveness of bright light therapy on depression, accompanied by improved sleep quality and internal biological rhythm. Bright light therapy (pale blue light at 7,500 lux for one hour) significantly decreased depressive symptoms compared with the placebo control group (red dim light at 50 lux) among 89 patients with non-seasonal depression, and was accompanied by an increase in salivary melatonin in the evening and better actigraphmeasured sleep parameters [22,23]. An intervention to increase light exposure by installing a ceiling light in the shared living room in group care facilities significantly decreased depressive symptoms, and increased total sleep time, as assessed by an actigraph, among elderly participants. In addition, a significant interaction of light intervention and melatonin administration on sleep efficiency was observed [24]. Higher daylight exposure increases melatonin secretion at night, according to an interventional study [25] and our population-based observational study [26].

Short-term and long-term influence of cataract surgery on depression and subjective sleep quality have been 
reported by some observational studies. Compared with depressive symptoms assessed before cataract surgery, significant decreases in depressive symptoms have reported at two months [27,28], three months [29], and one year [30] after surgery. In addition, improved subjective sleep quality was reported at one month [31], two months [32,33], and nine months [34] after surgery. However, evidence from RCTs on the effect of cataract surgery on depressive symptoms, sleep disturbance, and internal biological rhythms is lacking.

In contrast to bright light therapy conducted during the daytime, cataract surgery may increase the input of light information, not only in the daytime, but also during the night-time. Increased input of light information during the night-time may be deleterious to health outcomes. Indeed, we found significant cross-sectional association of increased light at night with increased prevalence of obesity, dyslipidaemia [35], sleep disturbance [36], and depression [37] in real-life situations among elderly individuals. These associations are supported by previous experimental evidence [38-40].

Here, we will conduct a parallel-group, assessorblinded, simple RCT comparing the intervention group at three months after surgery with the control group at three months after baseline, to determine whether cataract surgery modifies the internal biological rhythm and improves its related health outcomes such as depression, sleep quality, body mass regulation, and glucose and lipid metabolism.

\section{Methods/Design}

All intervention processes associated with the present study are conducted at Nara Medical University hospital. The protocol of this study has been registered at the University Hospital Medical Information Network Clinical Trials Registry (UMIN-CTR, identifier: UMIN000014559). The CLOCK-IOL (Cataract Surgery and Circadian Biological Rhythm among Japanese Older People with Cataract in Nara, Kansai Region: Influence of Intra Ocular Lens Implantation) study was approved by the institutional review board of Nara Medical University (approval number: 13-032). This research complies with the Declaration of Helsinki. Before enrolment to this study, we will obtain written informed consent from all participants.

We will include participants continuously through the year with similar speed, and inclusion of the present study will finish at the same date as the started year. After baseline measurements, all participants will be randomly allocated to either the intervention group or the control group in a 1:1 ratio without restrictions such as blocking and stratification. The outcomes among the intervention group will be measured at three months after surgery. Among the control group, the outcomes will be measured at three months after baseline. The control group will receive delayed cataract surgery shortly after the outcome measurement (Figure 1).

\section{Participants}

We will assess the eligibility of patients in Nara Medical University Hospital who have been diagnosed as having cataracts according to the following inclusion and exclusion criteria.

\section{Inclusion criteria}

The inclusion criteria for this study are as follows:

1. Patients scheduled for their first cataract surgery,

2. Aged 60 years or over, and

3. Diagnosed with cataracts of grade 2 nuclear opacification as defined by the lens opacities classification system III [41].

\section{Exclusion criteria}

The exclusion criteria for this study are as follows:

1. Major depression with current therapy,

2. Severe mental illness or dementia,

3. Severe corneal opacities with difficulty in assessment of lens opacity or fundal examination,

4. Glaucoma with a visual field deficit of at least mean deviation $>14 \mathrm{~dB}$ (Humphrey perimeter),

5. Vitreous haemorrhage,

6. Proliferative diabetic retinopathy,

7. Macular edema,

8. Age-related macular degeneration,

9. Patients needing immediate cataract surgery, or

10. Patients needing combined cataract and glaucoma surgery or combined cataract surgery and vitrectomy.

\section{Intervention}

Before the cataract surgery, the axial length of the eye will be measured with an A-scan UD-6000 (Tomey, Nagoya, Japan). Yellow aspherical IOL (SN60WF, Alcon, Fort Worth, Texas, United States), yellow spherical IOL (SN60AT, Alcon, Fort Worth, Texas, United States), or clear spherical IOL (SA60AT, Alcon, Fort Worth, Texas, United States) will be used for the cataract surgery. The appropriate power of the IOL will be estimated using the SRK/T formula for each IOL [42]. The kind of IOL used for the cataract surgery was randomly allocated to yellow spherical IOL, yellow aspherical IOL, or clear spherical IOL in a 1:1:2 ratio. After phacoemulsification with a small incision, IOL will be implanted.

\section{Patients of cataract surgery for both eyes}

We include participants undergoing cataract surgery for single and both eyes. The surgery for both eyes among the intervention group will be completed within the same 


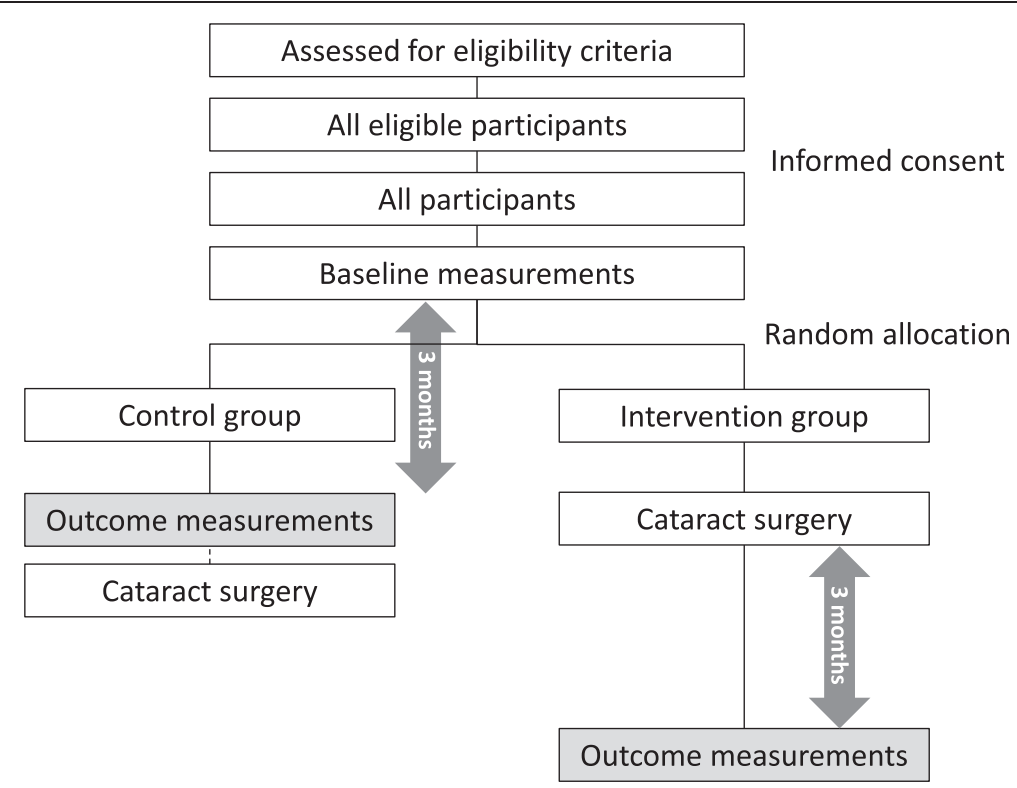

Figure 1 Flow chart of participants.

time-scale, one to two weeks, and the same kind of IOLs will be used for both eyes. Outcomes will be measured at three months after the latest surgery.

\section{Primary outcome}

We will assess depressive symptoms as a primary outcome of the present study using the short version geriatric depression scale GDS-15. Prevalence of depression, median of GDS-15, and the mean value of difference between baseline and three months later will be compared between the intervention group and the control group.

\section{Secondary outcomes}

As secondary outcomes, we will measure subjective and actigraph-measured sleep quality, sleepiness, glycated haemoglobin (HbA1c), fasting plasma glucose (FPG), triglyceride (TG), low-density lipoprotein cholesterol (LDL-C), high-density lipoprotein cholesterol (HDL-C), body mass index (BMI), abdominal circumference, circadian rhythm of physical activity and wrist skin temperature, urinary melatonin metabolite, chronotype, post-illumination pupil response (PIPR), visual acuity, and subjective visual function.

\section{Self-reported questionnaires}

Depressive symptoms are measured using GDS-15; a self-administered questionnaire consisting of 15 items [43]. The sensitivity and specificity of GDS-15 compared with diagnosis according to the Diagnostic and Statistical Manual of Mental Disorders (fourth edition) was 92.7\% and $54.8 \%$ with a cut-off point of four out of five, and $84.8 \%$ and $67.7 \%$ with a cut-off point of six out of seven, respectively [44]. According to a meta-analysis about the validity of GDS-15, the mean sensitivity was 0.805 and the mean specificity was 0.750 , respectively [45]. A higher score on the GDS-15 was significantly associated with self-report and clinician-administered measures of suicidal ideation [46] and higher rates of suicide [47]. Subjective sleep quality and daytime sleepiness are assessed using the Pittsburgh Sleep Quality Index (PSQI) [48] and the Epworth Sleepiness Scale (ESS) [49,50], respectively. Chronotype and subjective visual function are determined by the 'morningness-eveningness' questionnaire (MEQ) [51], the Munich Chronotype Questionnaire (MCTQ) [52], and the National Eye Institute Visual Function Questionnaire (NEI VFQ25) [53,54].

\section{Analysis of venous blood sample}

Overnight fasting venous blood samples will be analysed at a commercial laboratory (SRL Co. Inc., Tokyo, Japan) using standard clinical chemistry analysis to determine the concentrations of HbA1c, FPG, TG, LDL-C, and HDL-C.

\section{Morning spot urine}

We will measure 6-sulfatoxymelatonin (aMT6-s) in a morning spot urine sample. Peak nocturnal plasma melatonin is significantly associated with aMT6-s in subsequent morning spot urine $(\mathrm{r}=0.69)[55,56]$. Urinary aMT6-s concentration will be measured at a commercial laboratory (SRL Co. Inc. Tokyo, Japan) using an ELISA kit (RE54031; IBL International, Hamburg, Germany).

\section{Actigraph}

Objective sleep will be measured by an actigraph (ActiSleepBT Monitor; ActiGraph Inc., Florida, United States), worn 
on the non-dominant arm for five days, including weekdays and a weekend. Participants will be instructed to keep a standardised sleep diary logging bed time and rising time. Sleep parameters such as total sleep time, sleep efficiency, sleep onset latency, and wake after sleep onset will be calculated with ActiLife 6 (ActiGraph Inc. Florida, USA). A validation study of this device showed moderate to high agreement with sleep parameters as measured by polysomnography [57].

\section{Circadian rhythm of physical activity and wrist skin temperature}

Invasively measured biomarkers usually used in laboratory settings, such as fluctuation of plasma cortisol, melatonin, and rectal temperature, are difficult to conduct because they disturb the normal life of participants. To assess the influence of cataract surgery on internal biological rhythms using non-invasive methods, we will measure the phase and amplitude of circadian activity rhythm and the wrist skin temperature.

According to large-scale prospective cohort studies, decreased amplitude, later phase, and decreased robustness of circadian activity rhythm showed a significantly higher hazard ratio for incidents of cognitive disorders, cancer mortality, and all-cause mortality [58-60]. Wrist temperature reveals heat loss from arteriovenous anastomoses at the skin [61], and shows a mirror image of core body temperature [62] and blood pressure [63,64]. The validity of using wrist skin temperature as an acceptable measure to assess circadian phase is indicated by the significant correlation of dim light melatonin onset and the increase of wrist skin temperature in the evening in reallife situations $(r=0.76)[65]$. The wrist skin temperature at inside of the wrist, near the radial artery of the dominant arm, will be measured using a temperature data logger (Thermochron iButton; Maxim/Dallas, Dallas, Texas, United States) at three-minute intervals [62].

\section{Post-illumination pupil response}

By pharmacological blockage of the rod and cone cells, the melanopsin-associated ganglion cell response is isolated as a slow, maintained depolarisation to light stimulation, which repolarises slowly after light-offset in vitro [66]. This PIPR is indicated as an index of the sensitivity of the melanopsin-containing ipRGC pathway [67]. In the present study, baseline and sustained pupil diameter after blue and red light stimulation will be measured. Baseline pupil diameter is the average pupil diameter in a seven-second period before light onset. Sustained pupil diameter is the average from 10 to 40 seconds after light offset. PIPR (mm), PIPR change (\%), net PIPR ( $\mathrm{mm})$, and net PIPR change (\%) will be calculated as follows [68]:

$$
\begin{aligned}
\operatorname{PIPR}(\mathrm{mm})= & \text { Baseline pupil diameter }(\mathrm{mm}) \\
& - \text { Sustained pupil diameter }(\mathrm{mm})
\end{aligned}
$$

$\begin{aligned} \text { PIPR change }(\%)= & (\mathrm{PIPR} / \text { Baseline pupil diameter }) \\ & \times 100\end{aligned}$

Net PIPR $(\mathrm{mm})=$ Blue PIPR-Red PIPR

Net PIPR change $(\%)=$ Blue PIPR change $(\%)$ -Red PIPR change (\%)

\section{Other variables}

Using a standardised questionnaire, trained researchers will interview participants about basic characteristics, such as age, gender, smoking and drinking habits, past history of cardiovascular events, stroke, cancer, medication, household income, years of education, and history of shift work. BMI will be calculated as weight per height $\left(\mathrm{kg} / \mathrm{m}^{2}\right)$. Waist circumference will be measured at the level of the umbilicus, in the standing position.

\section{Sample size}

According to the HEIJO-KYO study [26], a communitybased cohort study among the elderly (mean age \pm standard deviation: $72 \pm 7.1$ ) in the same district as the present study, the prevalence of depression was $20.0 \%$ (101 out of 506) [37]. To detect its $7 \%$ difference as significant at a two-sided $\alpha$ level of $5 \%$ with a power of $80 \%$, it was calculated that 475 participants in each group would be required. Assuming a dropout of $5 \%$, we estimated that a total of 1,000 participants would be needed.

\section{Randomization and masking}

Allocation concealment will be maintained by central randomization using computer-generated random sequences, independent of care-providers. The results of the allocation will be masked from the assessors of the outcomes, but will be available to both the care providers and the participants.

\section{Statistical analysis}

We will compare the outcomes between the control group and the intervention group based on the intention-to-treat principle. For missing values due to loss to follow-up after baseline measurement, we will impute baseline data using the last observation carried forward (LOCF) method. For continuous variables with normal distributions, the mean and standard deviation will be reported. For variables not distributed normally, the median and interquartile range will be reported. Means, medians, and proportions will be compared using the $t$ test, the Mann-Whitney $U$ test, and the $\chi^{2}$ test, respectively. We will use analysis of covariance to estimate adjusted mean values and $95 \%$ CIs. The prevalence of the two groups will be tested using multivariate logistic regression analysis. To assess the phase, amplitude, and robustness of the circadian rhythm, we will use the cosinor model [69], the sigmoidally transformed cosine 
model [70], and the generalised additive model [71]. We will conduct a subgroup analysis at baseline according to the severity of cataract and chronotype.

\section{Discussion}

To conduct an RCT assessing the long-term effect of cataract surgery is ethically difficult, because the effectiveness of cataract surgery for visual acuity is established. To assess the effect of cataract surgery after three months with minimum disadvantage to the control group due to delayed surgery, we have potential limitations due to the difference of duration from baseline survey to outcome measurements between the two groups. Compared with the control group, the intervention group will spend a longer time from baseline measurement to outcome measurement due to the duration between baseline and surgery, and it may distort the results because of a seasonal effect on outcomes. However, we will continuously include participants with a similar speed though the year, and will finish this study at the same date as started in the previous year. As a result, the number of overestimated outcomes and underestimated outcomes due to the seasonal influence will be balanced between the two groups. In the statistical analysis, we will compare the outcomes adjusted for seasonal variables, such as day length at outcome measurement, using ANOVA for continuous variables and multivariate logistic regression analysis for categorical outcomes.

\section{Trial status}

At the time of submission, the study protocol has been fixed and registered for clinical trial registration, but the recruitment of participants has not started. Recruitment of participants is expected to begin August 2014.

\section{Abbreviations}

aMT6-s: 6-sulfatoxymelatonin; BMl: Body mass index; FPG: Fasting plasma glucose; GDS-15: The short version geriatric depression scale; HDL-C: High-density lipoprotein cholesterol; IOL: Intraocular lens; ipRGCs: Intrinsically photosensitive retinal ganglion cells; LDL-C: Low-density lipoprotein cholesterol; PIPR: post-illumination pupil response; RCTs: Randomized controlled trials; SCN: Suprachiasmatic nuclei; TG: Triglyceride.

\section{Competing interests}

The authors declare that they have no competing interests.

\section{Authors' contributions}

$\mathrm{KS}, \mathrm{KO}$, and TN initiated the study design. KS prepared the first draft of the manuscript. KM, SM, TU, MO, TH, and TM provided the ophthalmic expertise in this clinical trial. NT provided contributions from the engineering aspect for measuring outcomes. NK and NO are grant holders. All authors contributed to the refinement of study protocol and approved the final manuscript.

\section{Acknowledgments}

The present study is supported by a grant for collaboration study from Nara Medical University.

\section{Author details}

'Department of Community Health and Epidemiology, Nara Medical University School of Medicine, 840 Shijocho, Kashiharashi, Nara 634-8521, Japan. ${ }^{2}$ Department of Ophthalmology, Nara Medical University School of Medicine, 840 Shijocho, Kashiharashi, Nara 634-8521, Japan. ${ }^{3}$ Center for
Academic Industrial and Governmental Relations, Nara Medical University School of Medicine, 840 Shijocho, Kashiharashi, Nara 634-8521, Japan.

Received: 15 July 2014 Accepted: 10 December 2014

Published: 29 December 2014

References

1. Czeisler C, Duffy J, Shanahan T, Brown E, Mitchell J, Rimmer D, Ronda J, Silva E, Allan J, Emens J, Dijik DJ, Kronauer RE: Stability, precision, and near-24-hour period of the human circadian pacemaker. Science 1999, 284:2177-2181.

2. Flo E, Pallesen S, Akerstedt T, Mageroy N, Moen BE, Gronli J, Nordhus IH, Bjorvatn B: Shift-related sleep problems vary according to work schedule. Occup Environ Med 2013, 70:238-245.

3. Bara A-C, Arber S: Working shifts and mental health - findings from the British Household Panel Survey (1995-2005). Scand J Work Environ Health 2009, 35:361-367.

4. Karsson B, Karsson A, Lindahl B: Is there an association between shift work and having a metabolic syndrome? Results from a population based study of 27485 people. Occup Environ Med 2001, 58:747-752.

5. Pietroiusti A, Neri A, Somma G, Coppeta L, lavicoli I, Bergamaschi A, Magrini A: Incidence of metabolic syndrome among night-shift healthcare workers. Occup Environ Med 2010, 67:54-57.

6. Kroenke $\mathrm{CH}$, Spiegelman D, Manson J, Schernhammer ES, Colditz GA, Kawachi I: Work characteristics and incidence of type 2 diabetes in women. Am J Epidemiol 2007, 165:175-183.

7. Morikawa Y, Nakagawa H, Miura K, Soyama Y, Ishizaki M, Kido T, Naruse Y, Suwazono $Y$, Nogawa $K$ : Shift work and the risk of diabetes mellitus among Japanese male factory workers. Scand J Work Environ Health 2005, 31:179-183.

8. Vyas MV, Garg AX, lansavichus AV, Costella J, Donner A, Laugsand LE, Janszky I, Mrkobrada M, Parraga G, Hackam DG: Shift work and vascular events: systematic review and meta-analysis. BMJ 2012, 345:e4800.

9. Fujino $Y$, Iso $H$, Tamakoshi A, Inaba $Y$, Koizumi A, Kubo T, Yoshimura T, Group. JCCS: A prospective cohort study of shift work and risk of ischemic heart disease in Japanese male workers. Am J Epidemiol 2006, 164:128-135.

10. Brown DL, Feskanich D, Sanchez BN, Rexrode KM, Schernhammer ES, Lisabeth LD: Rotating night shift work and the risk of ischemic stroke. Am J Epidemiol 2009, 169:1370-1377.

11. Khalsa SB, Jewett ME, Cajochen C, Czeisler CA: A phase response curve to single bright light pulses in human subjects. J Physiol 2003, 549:945-952

12. Pulivarthy SR, Tanaka N, Welsh DK, De Haro L, Verma IM, Panda S: Reciprocity between phase shifts and amplitude changes in the mammalian circadian clock. Proc Natl Acad Sci U S A 2007, 104:20356-20361.

13. Hattar S, Liao HW, Takao M, Berson DM, Yau KW: Melanopsin-containing retinal ganglion cells: architecture, projections, and intrinsic photosensitivity. Science 2002, 295:1065-1070.

14. Brainard GC, Hanifin JP, Greeson JM, Byrne B, Glickman G, Gerner E, Rollag MD: Action spectrum for melatonin regulation in humans: evidence for a novel circadian photoreceptor. J Neurosci 2001, 21:6405-6412.

15. Mriotti SP: Global data on visual impairments. World Health Organ 2014, 2010:1-14.

16. Kessel L, Lundeman JH, Herbst K, Andersen TV, Larsen M: Age-related changes in the transmission properties of the human lens and their relevance to circadian entrainment. J Cataract Refract Surg 2010, 36:308-312.

17. Eramudugolla R, Wood J, Anstey KJ: Co-morbidity of depression and anxiety in common age-related eye diseases: a population-based study of 662 adults. Front Aging Neurosci 2013, 5:56.

18. Kessel $L$, Siganos $G$, Jorgensen $T$, Larsen M: Sleep disturbances are related to decreased transmission of blue light to the retina caused by lens yellowing. Sleep 2011, 34:1215-1219.

19. Turner PL, Van Someren EJ, Mainster MA: The role of environmental light in sleep and health: effects of ocular aging and cataract surgery. Sleep Med Rev 2010, 14:269-280.

20. Tuunainen A, Kripke DF, Endo T: Light therapy for non-seasonal depression. Cochrane Database Syst Rev 2004, CD004050.

21. Golden RN, Gaynes BN, Ekstrom RD, Hamer RM, Jacobsen FM, Suppes T, Wisner KL, Nemeroff CB: The efficacy of light therapy in the treatment of mood disorders: a review and meta-analysis of the evidence. Am J Psychiatry 2005, 162:656-662. 
22. Lieverse R, Nielen MM, Veltman DJ, Uitdehaag BM, van Someren EJ, Smit JH, Hoogendijk WJ: Bright light in elderly subjects with nonseasonal major depressive disorder: a double blind randomised clinical trial using early morning bright blue light comparing dim red light treatment. Trials 2008 9:48.

23. Lieverse R, Van Someren EJ, Nielen MM, Uitdehaag BM, Smit JH, Hoogendijk WJ: Bright light treatment in elderly patients with nonseasonal major depressive disorder: a randomized placebo-controlled trial. Arch Gen Psychiatry 2011, 68:61-70.

24. der Lek RF R-v, Swaab DF, Twisk J, Hol EM, Hoogendijk WJ, Van Someren EJ: Effect of bright light and melatonin on cognitive and noncognitive function in elderly residents of group care facilities: a randomized controlled trial. Jama 2008, 299:2642-2655.

25. Mishima K, Okawa M, Shimizu T, Hishikawa Y: Diminished melatonin secretion in the elderly caused by insufficient environmental illumination. J Clin Endocrinol Metab 2001, 86:129-134.

26. Obayashi K, Saeki K, Iwamoto J, Okamoto N, Tomioka K, Nezu S, Ikada Y, Kurumatani N: Positive effect of daylight exposure on nocturnal urinary melatonin excretion in the elderly: a cross-sectional analysis of the HEIJO-KYO study. J Clin Endocrinol Metab 2012, 97:4166-4173.

27. Gray CS, Karimova G, Hildreth AJ, Crabtree L, Allen D, O'Connell JE: Recovery of visual and functional disability following cataract surgery in older people: Sunderland Cataract Study. J Cataract Refract Surg 2006, 32:60-66.

28. Ishii K, Kabata T, Oshika T: The impact of cataract surgery on cognitive impairment and depressive mental status in elderly patients. Am J Ophthalmol 2008, 146:404-409.

29. Fraser ML, Meuleners LB, Lee AH, Ng JQ, Morlet N: Vision, quality of life and depressive symptoms after first eye cataract surgery. Psychogeriatrics 2013, 13:237-243

30. Meuleners LB, Hendrie D, Fraser ML, Ng JQ, Morlet N: The impact of first eye cataract surgery on mental health contacts for depression and/or anxiety: a population-based study using linked data. Acta Ophthalmol 2013, 91:e445-e449.

31. Asplund R, Ejdervik Lindblad B: The development of sleep in persons undergoing cataract surgery. Arch Gerontol Geriatr 2002, 35:179-187.

32. Wei $X$, She C, Chen D, Yan F, Zeng J, Zeng L, Wang L: Blue-light-blocking intraocular lens implantation improves the sleep quality of cataract patients. J Clin Sleep Med 2013, 9:741-745.

33. Ayaki M, Negishi K, Tsubota K: Rejuvenation effects of cataract surgery with UV blocking intra-ocular lens on circadian rhythm and gait speed. Rejuvenation Res 2014, 17:359-365.

34. Asplund R, Lindblad BE: Sleep and sleepiness 1 and 9 months after cataract surgery. Arch Gerontol Geriatr 2004, 38:69-75.

35. Obayashi K, Saeki K, Iwamoto J, Okamoto N, Tomioka K, Nezu S, Ikada Y, Kurumatani N: Exposure to light at night, nocturnal urinary melatonin excretion, and obesity/dyslipidemia in the elderly: a cross-sectional analysis of the HEIJO-KYO study. J Clin Endocrinol Metab 2013, 98:337-344.

36. Obayashi K, Saeki K, Kurumatani N: Association between light exposure at night and insomnia in the general elderly population: the HEIJO-KYO cohort. Chronobiol Int 2014, 31:976-982.

37. Obayashi K, Saeki K, Iwamoto J, Ikada Y, Kurumatani N: Exposure to light at night and risk of depression in the elderly. J Affect Disord 2013, 151:331-336.

38. Cajochen C, Zeitzer JM, Czeisler CA, Dijk DJ: Dose-response relationship for light intensity and ocular and electroencephalographic correlates of human alertness. Behav Brain Res 2000, 115:75-83.

39. Fonken LK, Kitsmiller E, Smale L, Nelson RJ: Dim nighttime light impairs cognition and provokes depressive-like responses in a diurnal rodent. J Biol Rhythms 2012, 27:319-327.

40. Fonken LK, Workman JL, Walton JC, Weil ZM, Morris JS, Haim A, Nelson RJ: Light at night increases body mass by shifting the time of food intake. Proc Natl Acad Sci U S A 2010, 107:18664-18669.

41. Chylack LT Jr, Wolfe JK, Singer DM, Leske MC, Bullimore MA, Bailey IL, Friend J, McCarthy D, Wu SY: The Lens Opacities Classification System III. The Longitudinal Study of Cataract Study Group. Arch Ophthalmol 1993, 111:831-836.

42. Sanders DR, Retzlaff J, Kraff MC: Comparison of empirically derived and theoretical aphakic refraction formulas. Arch Ophthalmol 1983, 101:965-967.

43. Burke WJ, Roccaforte WH, Wengel SP: The short form of the Geriatric Depression Scale: a comparison with the 30-item form. J Geriatr Psychiatry Neurol 1991, 4:173-178.
44. Almeida OP, Almeida SA: Short versions of the geriatric depression scale: a study of their validity for the diagnosis of a major depressive episode according to ICD-10 and DSM-IV. Int J Geriatr Psychiatry 1999, 14:858-865.

45. Wancata J, Alexandrowicz R, Marquart B, Weiss M, Friedrich F: The criterion validity of the Geriatric Depression Scale: a systematic review. Acta Psychiatr Scand 2006, 114:398-410.

46. Heisel MJ, Flett GL, Duberstein PR, Lyness JM: Does the geriatric depression scale (GDS) distinguish between older adults with high versus low levels of suicidal ideation? Am J Geriatr Psychiatry 2005, 13:876-883.

47. Sun WJ, Xu L, Chan WM, Lam TH, Schooling CM: Depressive symptoms and suicide in 56,000 older Chinese: a Hong Kong cohort study. Soc Psychiatry Psychiatr Epidemiol 2012, 47:505-514.

48. Buysee DJ, Reynolds CF III, Monk TH, Berman SR, Kupfer DJ: The Pittsburgeh Sleep Quality Index: a new instrument for psychiatric practice and research. Psychiatry Res 1988, 28:193-213.

49. Johns MW: Sleepiness in different situations measured by the Epworth Sleepiness Scale. Sleep 1994, 17:703-710.

50. Johns MW: Reliability and factor analysis of the Epworth Sleepiness Scale. Sleep 1992, 15:376-381.

51. Horne JA, Ostberg O: A self-assessment questionnaire to determine morningness-eveningness in human circadian rhythms. Int J Chronobiol 1976, 4:97-110.

52. Kitamura S, Hida A, Aritake S, Hiquchi S, Enomoto M, Kato M, Vetter C Roenneberg T, Mishima K: Validity of the Japanese version of the Munich ChronoType Questionnaire. Chronobiol Int 2014, 31:845-850.

53. Mangione CM, Lee PP, Gutierrez PR, Spritzer K, Berry S, Hays RD: Development of the 25-item National Eye Institute Visual Function Questionnaire. Arch Ophthalmol 2001, 119:1050-1058.

54. Suzukamo Y, Oshika T, Yuzawa M, Tokuda Y, Tomidokoro A, Oki K, Mangione CM, Green J, Fukuhara S: Psychometric properties of the 25-item National Eye Institute Visual Function Questionnaire (NEI VFQ-25), Japanese version. Health Qual Life Outcome 2005, 3:65.

55. Schernhammer ES, Rosner B, Wilett W, Laden F, Colditz G, Hankinson S: Epidemiology of urinary melatonin in women and its relation to other hormones and night work. Cancer Epidemiol Biomarkers Prev 2004 13:936-943.

56. Graham C, Cook M, Kavet R, Sastre A, Smith D: Prediction of nocturnal plasma melatonin form morning urinary measures. J Pineal Res 1998 24:230-238

57. Cellini N, Burman M, McDevitt E, Ricker A, Mednick S: Direct comparison of two actigraphy devices with polysomnographically recorded naps in healthy young adults. Chronobiology Int 2013, 30:691-698.

58. Tranah GJ, Blackwell T, Stone KL, Ancoli-Israel S, Paudel ML, Ensrud KE, Cauley JA, Redline S, Hillier TA, Cummings SR, Yaffe K, SOF Research Group: Circadian activity rhythms and risk of incident dementia and mild cognitive impairment in older women. Ann Neurol 2011, 70:722-732.

59. Tranah GJ, Blackwell T, Ancoli-Israel S, Paudel ML, Ensrud KE, Cauley JA Redline S, Hillier TA, Cummings SR, Stone KL: Circadian activity rhythms and mortality: the study of osteoporotic fractures. J Am Geriatr Soc 2010, 58:282-291.

60. Paudel ML, Taylor BC, Ancoli-Israel S, Stone KL, Tranah G, Redline S, Barrett-Connor E, Stefanick ML, Ensrud KE: Rest/activity rhythms and cardiovascular disease in older men. Chronobiol Int 2011, 28:258-266.

61. Krauchi K, Wirz-Justice A: Circadian rhythm of heat production, heart rate, and skin and core temperature under unmasking conditions in men. Am J Physiol 1994, 267:R819-R829.

62. Sarabia JA, Rol MA, Mendiola P, Madrid JA: Circadian rhythm of wrist temperature in normal-living subjects $A$ candidate of new index of the circadian system. Physiol Behav 2008, 95:570-580.

63. Krauchi K, Gompper B, Hauenstein D, Flammer J, Pfluger M, Studerus E, Schotzau A, Orgul S: Diurnal blood pressure variations are associated with changes in distal-proximal skin temperature gradient. Chronobiol Int 2012, 29:1273-1283.

64. Blazquez A, Martinez-Nicolas A, Salazar FJ, Rol MA, Madrid JA: Wrist skin temperature, motor activity, and body position as determinants of the circadian pattern of blood pressure. Chronobiol Int 2012, 29:747-756.

65. Bonmati-Carrion MA, Middleton B, Revell V, Skene DJ, Rol MA, Madrid JA: Circadian phase assessment by ambulatory monitoring in humans: correlation with dim light melatonin onset. Chronobiol Int 2014, 31:37-51.

66. Dacey DM, Liao HW, Peterson BB, Robinson FR, Smith VC, Pokorny J, Yau KW, Gamlin PD: Melanopsin-expressing ganglion cells in primate 
retina signal colour and irradiance and project to the LGN. Nature 2005, 433:749-754

67. Gamlin PD, McDougal DH, Pokorny J, Smith VC, Yau KW, Dacey DM: Human and macaque pupil responses driven by melanopsin-containing retinal ganglion cells. Vision Res 2007, 47:946-954.

68. Kankipati L, Girkin CA, Gamlin PD: Post-illumination pupil response in subjects without ocular disease. Invest Ophthalmol Vis Sci 2010, 51:2764-2769.

69. Barnett AG, Dobson AJ: Analysing Seasonal Health Data. Heidelberg: Springer; 2010.

70. Marler MR, Gehrman P, Martin UL, Ancoli-Israel S: The sigmoidally transformed cosine curve: a mathematical model for circadian rhythms with symmetric non-sinusoidal shapes. Stat Med 2006, 25:3893-3904.

71. Wood SN: Generalized Additive Models An Introduction with R. Boca Raton: Chapman and Hall/CRC; 2006.

doi:10.1186/1745-6215-15-514

Cite this article as: Saeki et al:: Short-term influence of cataract surgery

on circadian biological rhythm and related health outcomes (CLOCK-IOL trial): study protocol for a randomized controlled trial. Trials 2014 15:514.

\section{Submit your next manuscript to BioMed Central and take full advantage of:}

- Convenient online submission

- Thorough peer review

- No space constraints or color figure charges

- Immediate publication on acceptance

- Inclusion in PubMed, CAS, Scopus and Google Scholar

- Research which is freely available for redistribution 